\title{
Espondilectomia para sarcoma lombar primário de Ewing em crianças*
}

\section{Spondylectomy for Primary Ewing Lumbar Sarcoma in Children}

\author{
Carolina Oliveira10 Luísa Vital ${ }^{2}$ Francisco Serdoura ${ }^{2}$ André Rodrigues Pinho ${ }^{2}$ Vitorino Veludo ${ }^{2}$ \\ ${ }^{1}$ Departamento de Cirurgia Ortopédica, Unidade Local de Saúde do \\ Alto Minho, Viana do Castelo, Portugal \\ 2 Departamento de Cirurgia Ortopédica, Hospital de São João, Faculdade de \\ Endereço para correspondência Carolina Oliveira, Departamento de \\ Cirurgia Ortopédica, Unidade Local de Saúde do Alto Minho, Viana do \\ Castelo, Portugal (e-mail: carolinafernandesoliveira@gmail.com).
} Medicina, Universidade do Porto, Porto, Porto, Portugal

Rev Bras Ortop 2020;55(5):649-652.

\section{Resumo}

Palavras-chave

- neoplasias da coluna vertebral/cirurgia

- sarcoma de Ewing/ cirurgia

- vértebras lombares

- osteossarcoma/ cirurgia
O sarcoma de Ewing da coluna vertebral é raro, e seu tratamento é multidisciplinar. Não há consenso sobre o método ideal de controle local do tumor; no entanto, a ressecção em bloco com margens negativas está associada a uma melhora da sobrevida. Os autores relatam um caso de uma paciente de 5 anos do sexo feminino que inicialmente se apresentou com dor lombar, tendo sido diagnosticada com sarcoma de Ewing de acordo com o estudo imagiológico por radiografia, ressonância magnética e biópsia óssea. A paciente foi submetida a vertebrectomia após quimioterapia, de acordo com o protocolo Euro Ewing. Aos três anos de seguimento, não apresentou restrições nas atividades da vida diária, e, até o momento, não houve evidência de recidiva.
Abstract
Keywords
- spinal neoplasms/ surgery
- Ewing sarcoma/ surgery
- lumbar vertebrae
- osteosarcomal surgery

Primary Ewing sarcoma in the spine is very rare, and the treatment for it is multidisciplinary. There is no consensus regarding the optimal method of local control; however, en bloc resection is associated with an improvement in survival rates. The authors report a case of a 5-year-old girl who initially presented low back pain, and was diagnosed with Ewing sarcoma after being submitted to imaging studies by radiography, magnetic resonance and bone biopsy. A spondylectomy was performed in accordance with the Euro Ewing protocol. At the three-year follow-up, the patient had no restrictions regarding her daily activities, and there has been no evidence of recurrence to date.

\section{Introdução}

O sarcoma de Ewing foi descrito pela primeira vez por James Ewing em 1921, e é o segundo tumor ósseo maligno mais frequente em crianças, representando $3 \%$ dos tumores pediá-

\footnotetext{
Estudo conduzido no Departamento de Cirurgia Ortopédica, Hospital de São João, Faculdade de Medicina, Universidade do Porto, Porto, Portugal. Originalmente Publicado por Elsevier Editora Ltda.
}

recebido

06 de Setembro de 2017

aceito

19 de Outubro de 2017
DOI https://doi.org/

10.1016/j.rboe.2017.10.010. ISSN 0102-3616. tricos e $10 \%$ dos tumores ósseos primários. ${ }^{1} \mathrm{O}$ tumor normalmente acomete os ossos longos (em especial o fêmur) e a pelve. ${ }^{2-4} \mathrm{O}$ acometimento primário da coluna é raro, com incidência relatada de $5 \%$ de todos os sítios primários. ${ }^{2,3}$

Os sintomas mais comuns do sarcoma de Ewing são a dor localizada e o comprometimento neurológico, variando de radiculopatia a paraplegia. ${ }^{1,5,6}$

As técnicas de diagnóstico por imagem geralmente revelam destruição agressiva do corpo vertebral; a maioria dos pacientes apresenta uma massa extraóssea de tecido mole com invasão
Copyright $\odot 2020$ by Sociedade Brasileira License terms de Ortopedia e Traumatologia. Published by Thieme Revnter Publicações Ltda, Rio de Janeiro, Brazil 
direta das estruturas adjacentes. A ressonância magnética (RM) é o método de escolha para o estudo detalhado do envolvimento paravertebral. ${ }^{1,5}$

O tratamento cirúrgico ainda é desafiador. A ressecção em bloco geralmente é realizada, e está diretamente relacionada ao prognóstico. ${ }^{2,7}$

Nas últimas três décadas, a abordagem multidisciplinar e o tratamento combinado à quimioterapia e radioterapia contribuíram para a melhora da sobrevida desses pacientes. ${ }^{5,7}$

\section{Relato de Caso}

Uma menina de 5 anos foi atendida no Serviço de Emergência Pediátrica com dor lombar e abdominal, predominantemente à noite. Seus sintomas começaram um mês antes da consulta, com dor lombar progressiva e exacerbação nas últimas duas semanas. A dor não respondeu aos analgésicos. Os resultados do exame físico e dos exames laboratoriais não eram dignos de nota.

Uma radiografia da coluna lombar foi realizada na admissão, e revelou uma lesão lítica centrada em L1 (-Fig. 1). Uma ressonância magnética (RM) subsequente mostrou o colapso parcial do corpo vertebral de L1 devido a uma lesão infiltrativa com acometimento principalmente da metade posterior direita do corpo vertebral e do pedículo ipsilateral, associado a uma grande massa extraóssea de tecido mole com extensão intracanalar (-Fig. 2). As imagens sugeriram um processo agressivo. A paciente foi submetida a uma biópsia óssea de L1, e as características morfológicas e imunofenotípicas, à análise histológica, foram condizentes com um sarcoma de Ewing/tumor neuroectodérmico primitivo (PNET). A tomografia computadorizada (TC) de estadiamento não revelou a presença de metástases distantes.

Após a quimioterapia, realizada de acordo com o protocolo Euro Ewing, a paciente foi submetida ao tratamento cirúrgico. A espondilectomia foi realizada em dois estágios sob neuromonitoramento da medula espinhal por meio de potenciais evocados somatossensoriais e motores. Na primeira parte do procedimento, realizamos uma fixação pedicular de D11-L3 e a excisão dos elementos posteriores e

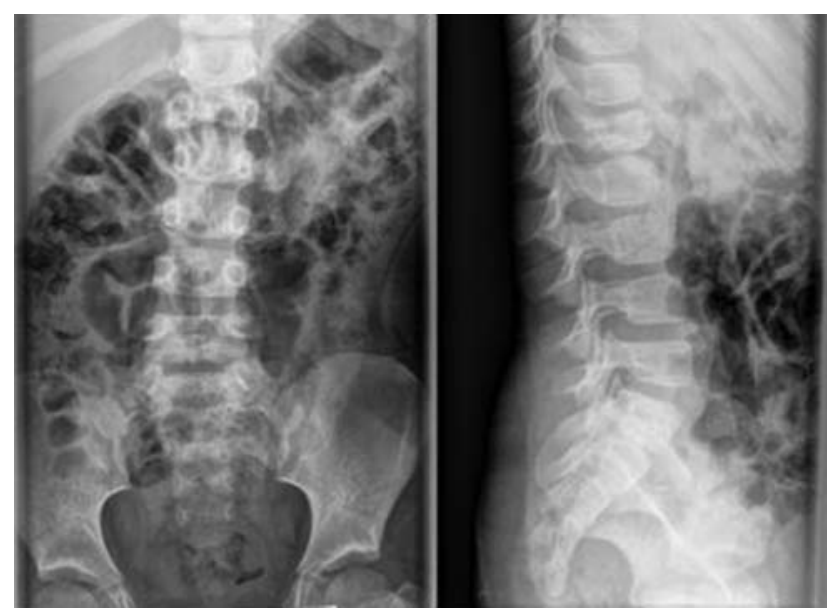

Fig. 1 A radiografia da coluna lombar (projeção anteroposterior e lateral) que identificou a lesão lítica em L1.

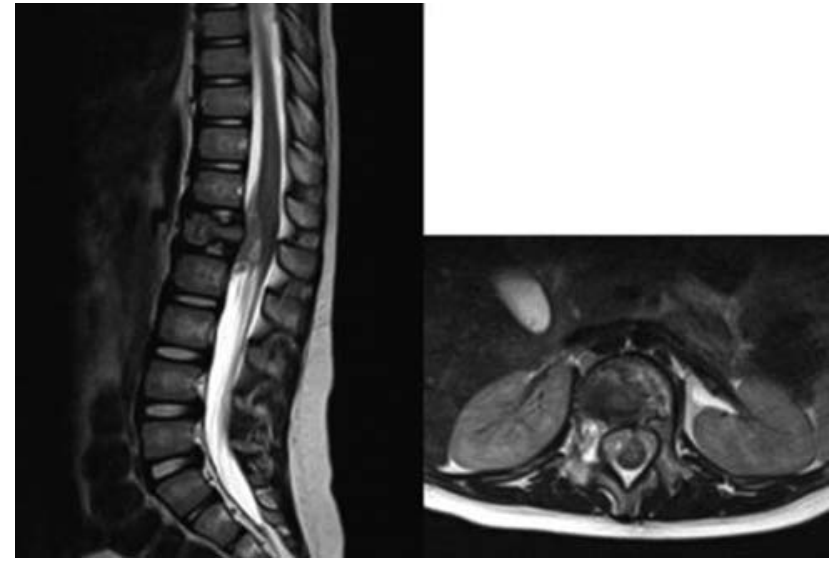

Fig. 2 Sequência de ressonância magnética ponderada em T2 com contraste da coluna lombar. Colapso de L1 por componente infiltrativo lítico com acometimento da metade posterior direita do corpo vertebral e da região pedicular ipsilateral, além de extensos componentes intracanalar e extramedular de tecido mole (Tomita et al. ${ }^{12}$ tipo 4).

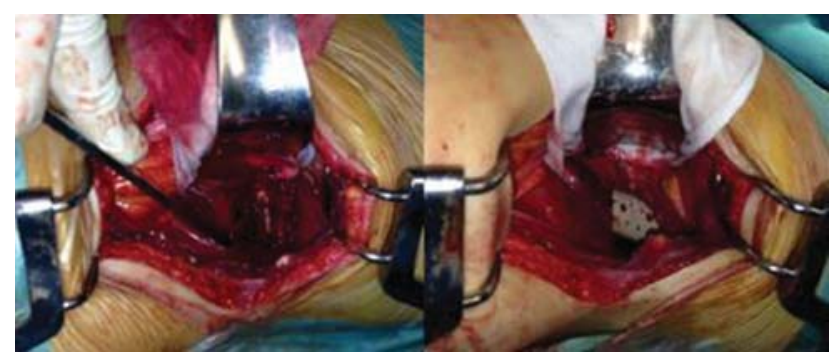

Fig. 3 Imagens intraoperatórias: à esquerda, após a espondilectomia; à direita, reconstrução anterior com gaiola.

pedículos de L1 por abordagem posterior. Em um segundo estágio, a corpectomia anterior e a reconstrução utilizando uma gaiola Medtronic (Mineápolis, MN, EUA) preenchida com enxerto ilíaco autólogo foi realizada por meio de uma abordagem anterior (-Fig. $\mathbf{3}$ ).

A paciente recebeu alta hospitalar 13 dias após a cirurgia, sem déficit neurológico. De acordo com o protocolo, continuou o tratamento adjuvante com quimioterapia e radioterapia por seis meses. Na primeira consulta de acompanhamento, apresentou retardo na cicatrização da ferida operatória com necrose das bordas, e houve necessidade de cuidados prolongados com curativos por quatro semanas. Não houve complicações com a instrumentação (-Fig. 4).

Durante um período de três anos de acompanhamento, a paciente não apresentou restrições às atividades diárias e, até o momento, não houve evidências de recidiva. Uma TC de acompanhamento revelou fusão dos níveis instrumentados (-Fig. 5).

\section{Discussão}

A coluna é frequentemente acometida em casos de sarcoma de Ewing metastático. No entanto, o sarcoma primário de Ewing na coluna é raro. ${ }^{4}$ Os achados de imagem são variáveis, mas a lesão lítica destrutiva com colapso parcial do corpo vertebral é o quadro mais comum. ${ }^{7,8}$ Manifestações sistêmicas, como febre e perda de peso, acompanhadas por 


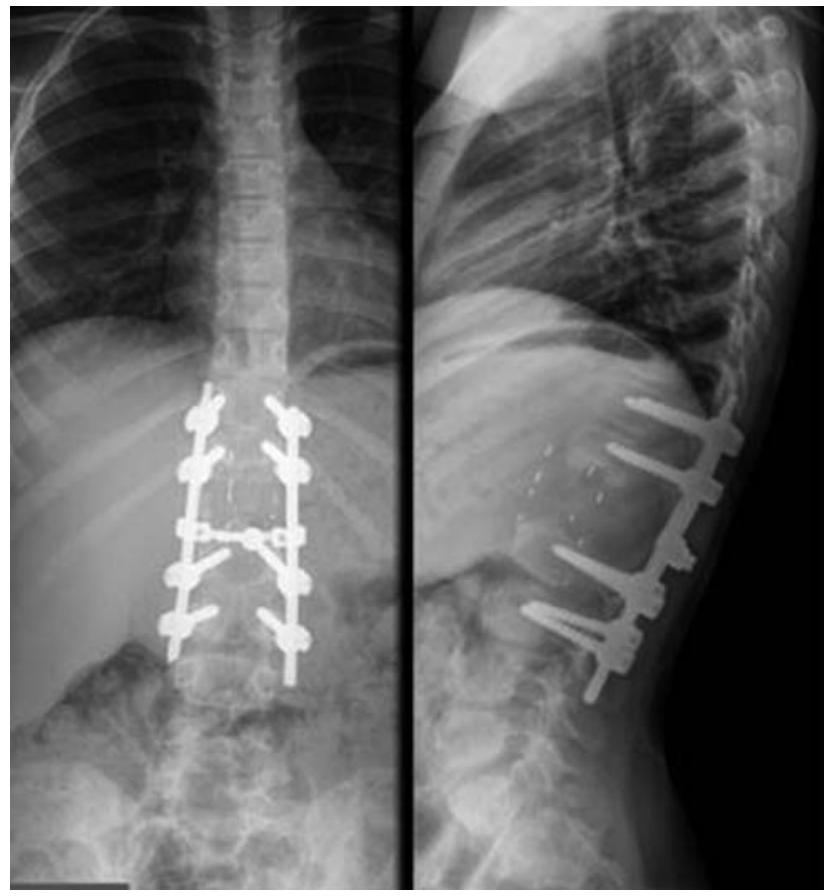

Fig. 4 Radiografia da coluna lombar (projeção anteroposterior e lateral) em um mês de acompanhamento.

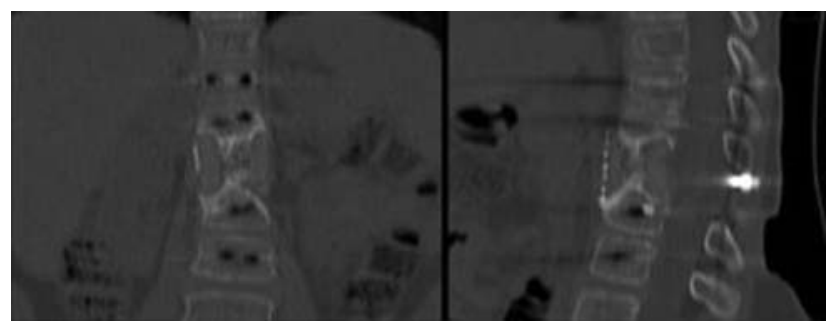

Fig. 5 A tomografia computadorizada realizada três anos após a espondilectomia mostra o crescimento ósseo pela gaiola.

leucocitose e aumento da sedimentação eritrocitária, podem ser observadas. ${ }^{1,7}$ Devido à variabilidade das características de imagem e ao fenótipo variável, o diagnóstico diferencial inclui infecção, neuroblastoma, granuloma eosinofílico e cisto ósseo aneurismático. ${ }^{7}$ A RM é fundamental na avaliação da extensão tumoral e no planejamento pré-operatório. ${ }^{1,4,8}$

O tratamento do sarcoma de Ewing é multidisciplinar. A maioria dos pacientes com diagnóstico inicial de doença localizada posteriormente apresenta micrometástases sistêmicas, o que enfatiza a necessidade de tratamento adjuvante em todos os indivíduos após o controle cirúrgico do tumor local. ${ }^{1}$ Marco et al. ${ }^{3}$ mostraram a maior incidência de recidiva tumoral e a menor taxa de sobrevida em pacientes tratados somente com quimioterapia e radioterapia em comparação aos resultados relatados por autores que realizaram a cirurgia agressiva. Por isso, a ressecção cirúrgica agressiva parece melhorar o prognóstico. ${ }^{3}$

A classificação de Tomita et al. ${ }^{12}$ baseada na localização anatômica dos tumores nos planos axial e sagital, orienta o tratamento cirúrgico. Neste caso, o tumor foi classificado como de tipo 4 , e o tratamento cirúrgico indicado foi a espondilectomia. $^{8}$
Ainda não há consenso sobre o método ideal de controle local do tumor. Atualmente, a ressecção em bloco com margens negativas é aceita como o tratamento ideal desses sarcomas, e está associada a uma melhora nas taxas de sobrevida. ${ }^{3,7,9}$ Uma revisão sistemática publicada por Sciubba et al. ${ }^{10}$ sugere que a ressecção cirúrgica agressiva está associada à melhora na taxa de sobrevida geral e no controle local. ${ }^{10}$

O objetivo da ressecção vertebral em bloco é permitir a retirada completa do tumor com margens livres e diminuir a probabilidade de recidiva. ${ }^{5,11}$ Tomita et al. ${ }^{12}$ não relataram recidiva local em um total de 23 pacientes submetidos a ressecção vertebral em bloco. ${ }^{12}$

Diferentes técnicas de ressecção em bloco foram descritas na literatura, como a técnica posterior ou as abordagens combinadas posterior e anterior. ${ }^{11}$ No entanto, a ressecção em bloco nem sempre é possível devido à proximidade do tumor com as estruturas nervosas.

No caso aqui descrito, os autores preferiram uma abordagem em dois estágios. A abordagem posterior permitiu a estabilização de dois níveis vertebrais acima e abaixo de L1 com parafusos pediculares, e a ressecção dos elementos posteriores de L1 com descompressão medular e liberação das raízes nervosas D12 e L1. No segundo estágio, mediante uma abordagem anterior, o corpo vertebral e a parte restante do pedículo foram removidos juntamente com discectomias completas de D12-L1 e L1-L2, com melhor controle local dos grandes vasos e vísceras adjacentes. Esta abordagem também permitiu a reconstrução da coluna anterior com uma gaiola expansível. Em um estudo biomecânico, Oda et al. ${ }^{13}$ descreveram que a instrumentação pedicular posterior associada à reconstrução anterior com gaiola proporcionou melhor estabilidade após espondilectomia. ${ }^{13}$

Como complicação, nossa paciente apresentou necrose das bordas da ferida cirúrgica, que já foi descrita por outros autores. ${ }^{11,14}$ Outras complicações frequentes descritas na literatura são problemas dos dispositivos metálicos, pseudoartrose ou infecção. ${ }^{11}$

Em conclusão, o sarcoma primário de Ewing na coluna é raro. $O$ tratamento dessas lesões ainda é um desafio, mas a ressecção em bloco do tumor seguida de tratamento adjuvante com radioterapia e quimioterapia está associada a um melhor prognóstico.

\section{Conflito de Interesses}

Os autores declaram não haver conflito de interesses.

\section{Referências}

1 Maheshwari AV, Cheng EY. Ewing sarcoma family of tumors. J Am Acad Orthop Surg 2010;18(02):94-107

2 Sewell MD, Tan KA, Quraishi NA, Preda C, Varga PP, Williams R. Systematic review of en bloc resection in the management of Ewing's sarcoma of the mobile spine with respect to local control and disease-free survival. Medicine (Baltimore) 2015;94(27): e1019

3 Marco RA, Gentry JB, Rhines LD, et al. Ewing's sarcoma of the mobile spine. Spine 2005;30(07):769-773

4 Feng H, Wang J, Guo P, Xu J, Feng J. Revision surgical treatment of a second lumbar Ewing Sarcoma: a report of a rare case. Medicine (Baltimore) 2015;94(30):e1190 
652 Espondilectomia para sarcoma lombar primário de Ewing em crianças Oliveira et al.

5 Sciubba DM, Hsieh P, McLoughlin GS, Jallo GI. Pediatric tumors involving the spinal column. Neurosurg Clin N Am 2008;19(01): 81-92

6 Fenoy AJ, Greenlee JD, Menezes AH, et al. Primary bone tumors of the spine in children. J Neurosurg 2006;105(04, Suppl):252-260

7 Akeda K, Kasai Y, Kawakita E, Seto M, Kono T, Uchida A. Primary Ewing sarcoma of the spine mimicking a psoas abscess secondary to spinal infection. Spine 2009;34(09):E337-E341

8 Kim HJ, McLawhorn AS, Goldstein MJ, Boland PJ. Malignant osseous tumors of the pediatric spine. J Am Acad Orthop Surg 2012;20(10):646-656

9 Rao G, Suki D, Chakrabarti I, et al. Surgical management of primary and metastatic sarcoma of the mobile spine. J Neurosurg Spine 2008;9(02):120-128
10 Sciubba DM, Okuno SH, Dekutoski MB, Gokaslan ZL. Ewing and osteogenic sarcoma: evidence for multidisciplinary management. Spine 2009;34(22, Suppl):S58-S68

11 Liljenqvist U, Lerner T, Halm H, Buerger H, Gosheger G, Winkelmann W. En bloc spondylectomy in malignant tumors of the spine. Eur Spine J 2008;17(04):600-609

12 Tomita K, Kawahara N, Baba H, Tsuchiya H, Fujita T, Toribatake Y. Total en bloc spondylectomy. A new surgical technique for primary malignant vertebral tumors. Spine 1997;22(03):324-333

13 Oda I, Cunningham BW, Abumi K, Kaneda K, McAfee PC. The stability of reconstruction methods after thoracolumbar total spondylectomy. An in vitro investigation. Spine 1999;24(16):1634-1638

14 Krepler P, Windhager R, Bretschneider W, Toma CD, Kotz R. Total vertebrectomy for primary malignant tumours of the spine. J Bone Joint Surg Br 2002;84(05):712-715 